\title{
Mouse exploration and choice of nestboxes differing in size
}

\author{
MARIE-CHRISTINE BUHOT \\ Laboratoire de Neurosciences Fonctionnelles, C.N.R.S., Marseille, France
}

\begin{abstract}
In this study, mice's preferences for nestboxes of various sizes were investigated by using, in social conditions, a progressive elimination procedure. Male and female groups (Experiment 1) preferred the medium-small nestbox $(15 \mathrm{~cm}$ in diameter) as a nest site, followed by the next smaller or next larger one; the largest boxes were chosen last. The order of initial exploration gave different results, with the mice ranking the nestboxes in this case by decreasing order of size. No systematic gender- or group-size-related effects were observed. Six groups of 2 male mice and four groups of 3 male mice (Experiment 2) were subjected to two elimination tests among nestboxes that differed in either both their inner and outer dimensions (matched condition) or their inner dimensions alone (mismatched condition). Except for the smallest nestbox, which was rejected by the mice, the order of preference for nestboxes was linear, in the direction of increasing size. No difference was observed between matched versus mismatched conditions. By contrast, the initial visits (and the number of visits) took the order of decreasing nestbox size, but only in the matched condition, in which the outer dimensions varied; in the mismatched condition, in which the nestboxes were identical on the outside, the various inner dimensions were not found to have any systematic effect on exploratory activity. These results are discussed in terms of the relevance of the spatial features, depending on the motivation (exploration or nest establishment) underlying behavior at a particular time.
\end{abstract}

Two basic biological functions underlie nest building and nest-site choice in mammals: (1) "to protect the animal from the extremes of heat and cold of the environment," and (2) "to provide a place in which to bear and raise its young" (Lisk, Pretlow, \& Friedman, 1969). Nest building is thus assumed to be a physiological behavioral complex (Lynch, 1973) that promotes the survival of an individual and the species it belongs to. The present study was not devoted to the study of nest building in itself but rather to the short-term aspect of nest-site choice and the circumstances that surround choice making. It focused especially on investigating which spatial properties of possible nest sites satisfy the animals' search for physical comfort, which is the most conspicuous motivation underlying nest establishment (Mulder, 1975).

Buhot-Averseng (1981) observed that mice, as individuals, first chose as their nest sites the smaller, rectangular ones among a set of nestboxes that differed in shape (rectangular, square, circular) and size (two modalities). When not seeking nest sites, the same subjects displayed the reverse tendency by exploring the larger nestboxes

\footnotetext{
The author is supported by the C.N.R.S. as "Chargée de Recherche." This research was also partly supported by a grant from Fondation de la Recherche Médicale Française to Henri Durup, Director of the Département de Psychologie Animale. Some of the data were presented at the C.E.E. meeting on "Social space in farm animals" (Organizer: René Zayan), Brussels, January 10-11, 1985. Henriette Lucchessi provided constant technical assistance during this research. The author's mailing address is Laboratoire de Neurosciences Fonctionnelles, C.N.R.S.-L.N.F. Ul bis, 31, chemin Joseph-Aiguier, 13402 Marseille cedex 9, France.
}

before they explored the smaller ones, whatever the shape. These results showed, in particular, that the use of size depended upon motivation: size was appraised differently depending on whether the mouse was engaged in exploration or in nest establishment.

The present study was designed to further investigate this hypothesis, using nestboxes that differed only in size, using various modalities along a scale consistent with that of the animal's familiar environment. The sets of nestboxes used here were chosen on the basis of the average sizes (10-15 cm in diameter) of a nest commonly built in the rearing conditions of our species. The aim of the present study was to compare experimentally a subjective (preferential) scale with an objective (metric) one (Buhot, 1986a).

In the present experiments, the subjects were not tested individually, as they had been previously, but in samesex cagemate groups of varying sizes. To compare isolation versus cagemate group testing conditions, this condition was used recently (Buhot, 1986b) with nestboxes that differed in design. It was shown there that individuals (of both sexes) used shape as the main choice criterion, whereas groups (male and female) preferentially took smallness of size into account. This social condition was used first to measure whether size, as the differentiating factor among the nestboxes, might be a relevant variable in social nest establishment and, secondly, to reduce the artificial component introduced by separating one animal from its familiar physical and social environment to test it alone in a new, unknown space. Moreover, the preferences expressed by groups composed of various familiar 
individuals for nestboxes differing in size are likely to constitute adaptive responses to group density requirements; that is, a positive correlation was to be expected between the size of the group and that of the preferred nestbox. The aim of this study was thus also to further investigate any sex-related nestbox-choice behavior. Male and female groups of different sizes were therefore subjected to Experiment 1 with a set of six simple nestboxes that differed in size.

\section{EXPERIMENT 1}

\section{Method}

Subjects. The animals used as subjects were 18 male and $21 \mathrm{fe}-$ male albino mice (Mus musculus L.) born in a closed outbred laboratory strain from the Noirot colony (E.N. strain, Brussels). They were provided with food and water ad lib and kept on a 12-h light/dark cycle that was maintained automatically $(6: 00$ a.m. to 6:00 p.m. being the light period). The breeding room and the adjacent testing room were maintained at $23^{\circ} \pm 2^{\circ} \mathrm{C}$. These rearing conditions were maintained until the end of the experiment. The mice had been reared since birth in circular Plexiglas cages, $34.9 \mathrm{~cm}$ in diameter and $16 \mathrm{~cm}$ in height. At weaning, they were placed in identical clean cages with their same-sex littermates. Twelve split litters, or groups (each containing 2 to 5 individuals), were thus formed, six of males and six of females. They were familiar with the experimental situation, since they had been previously subjected to a similar test but with quite different stimuli (nestboxes of various shapes). It is probable that this previous experience only reduced the novelty of the present situation, probably making it less shynessinducing, and did not affect the relevance of the results of this preliminary report. Other studies have shown that the effects of previous nest-building experience or repeated testing are relatively insignificant (Flitner Kinder, 1927; Lisk et al., 1969). The subjects were approximately 6 months old when finally tested.

Test situation and Stimuli (Figure 1). The apparatus used to test the subjects was a circular open field, $160 \mathrm{~cm}$ in diameter and $40 \mathrm{~cm}$ in height, made of sheet metal laid on the flagstone floor of the experimental room. The stimuli were six removable $8-\mathrm{cm}$ high nestboxes. They were made of sheet metal, were circularshaped with a $5-\mathrm{cm}$ aperture, and had no lids. They differed in size but were otherwise identical; their diameters were $7.5,10,15,20$,

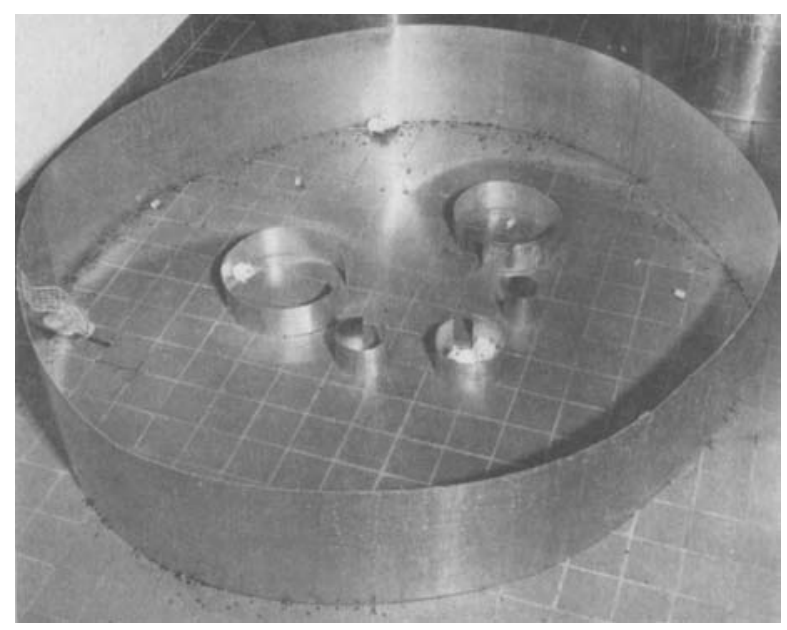

Figure 1. Experimental open field containing the remaining nestboxes after removal of the first nestbox to be chosen.
25 , and $30 \mathrm{~cm}$. These objects were arranged on the arena in a circle, with their apertures turned toward the center of the field. A square of cellulose for use as nesting material was placed in front of each aperture along with a bit of familiar food. A bottle of water was hung on the inner wall of the arena. The positions of this bottle, the nestboxes, and the testing order were set up pseudorandomly in order to avoid position cues and order effects.

Procedure. The experimental method was the continuous successive choice test using a progressive elimination procedure similar to that used previously (Buhot-Averseng, 1981). The experiment began around 9:00 a.m., with the arena clean and the nestboxes in place as described above. Two (or occasionally three) experimenters simultaneously placed in the middle of the field the mice from the group to be tested. During the first $30 \mathrm{~min}$ of the test, the observers noted all the entries (visits) to each of the nestboxes made by each mouse. About every hour after that, the animals' whereabouts were noted. When it was observed that a nestbox had been occupied for at least $2 \mathrm{~h}$ with a nest built inside, the first choice was considered to have been made and the experimenter withdrew the first nestbox chosen, causing the mice to move their nests toward another, still-available box; if the 2-h stability criterion was reached, this was recorded as the second choice; and so on, until only one nestbox was left. A complete preferential ranking was thus obtained. If, after the 48-h time limit, more than one nestbox still remained, so that the ranking was incomplete, the remaining boxes were assigned the average of the remaining ranks for the purpose of data analysis.

\section{Results}

Data analysis. The main data collected by the ranking procedure described above were the ranks assigned by the mice in terms of either successive nest sites (choice) or successively visited objects (exploratory patterns). In certain cases, other data were collected: the latency of the initial visit to each nestbox and the number of visits within the first 30 min of the test. Special ranking methods (Kendall, 1962; see also Buhot-Averseng, 1981, Appendix II) were used to analyze rankings, particularly the concordance between the groups as to their ranking of objects according to a particular criterion. Since the metric variable used here to differentiate the nestboxes was presented on a regular, progressive, and objective scale, the analysis on ranks of preference or on temporal order of exploration could reveal any existing correlation between the subjective scale and the objective one. Any concordance between groups would thus reveal to what extent the metric characteristics of the nextboxes were used as a common criterion. The effect of size was further analyzed by using nonparametric statistical procedures (Siegel, 1956) applied to the ranks allotted to the nestboxes.

Nestbox choice. Since the subjects were tested in six male and six female groups, the curves illustrating the results take this sex variable into account.

Figure 2, which shows the animals' choices of nestboxes as nest sites, indicates that the preferred nestbox (the optimum value) was the medium-small one $(15 \mathrm{~cm}$ in diameter). The next preferred nestbox was the nearest in size around this value $(10,20$, and $7.5 \mathrm{~cm})$. The two largest nestboxes were the last to be chosen. The values for the male and female groups overlap considerably, which means that no obvious sex-related effect was involved. Kendall's (1962) $W$ coefficient of concordance, cal- 


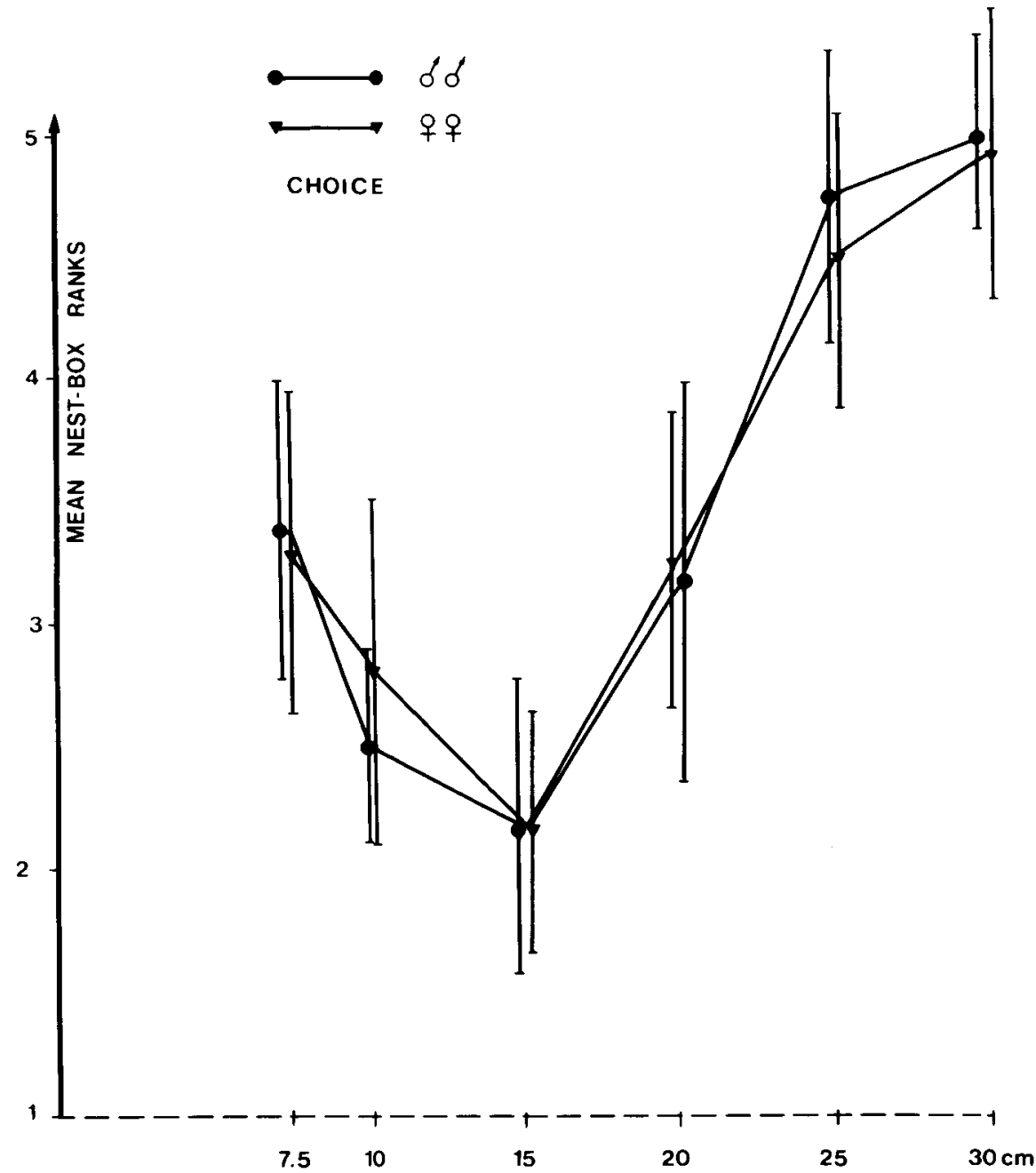

Figure 2. Nestbox choices in male and female groups. Mean $( \pm S E M)$ nestbox ranks (on the ordinate) of the six nestboxes that differed in size (diameter in centimeters on the horizontal axis). Standard errors are represented by vertical bars.

culated on the data of male and female groups combined, shows that whatever their sex, the groups strongly agreed in their preferences for size of nestbox $(S=1,081.75$, $W=0.429, \varrho=+0.377, z=1.05$, with $d f 1=5$ and $d f 2=50, p<.01)$.

To measure whether, apart from the optimum observed, any systematic preferences were accorded to the smaller nestboxes, the Wilcoxon matched-sign test was applied to the differences between the sum of ranks allotted to the three smallest nestboxes and the sum of ranks allotted to the three largest ones. It shows that, in fact, the groups of both sexes significantly chose smaller nestboxes before larger ones, thus indicating a consistent preference for the former $(T=0, N=12, p<.01$, two-tailed). No significant correlation between the sizes of the nestboxes and the sizes of the groups (both sexes combined) was observed when taking only the first choices into account $(r=-0.39$, n.s.); at most, a weak negative correlation was obtained.
Exploration patterns. The initial exploratory patterns were analyzed in terms of the order of the first visit to each of the six nestboxes by each group, regardless of which mouse actually entered the nestbox first.

Figure 3 shows the mean nestbox ranks for the male and the female groups separately. These curves can be seen to be negatively correlated with the sizes of the nestboxes (on the horizontal axis). This means that both the male and female groups visited the largest nestboxes first, and that the objects were then visited in decreasing order of size (except for the smallest one). Male and female data were pooled together to measure the conformity of exploratory behavior among the groups (since no obvious differences were found with regard to sex). Kendall's $W$ coefficient of concordance showed that the groups were significantly in agreement in visiting the nestboxes on the basis of a common rule, which, as shown in Figure 3, would seem to be to visit the nestboxes in decreasing order of size $(S=539, W=0.214, \mathrm{e}=+0.142, z=0.55$, 


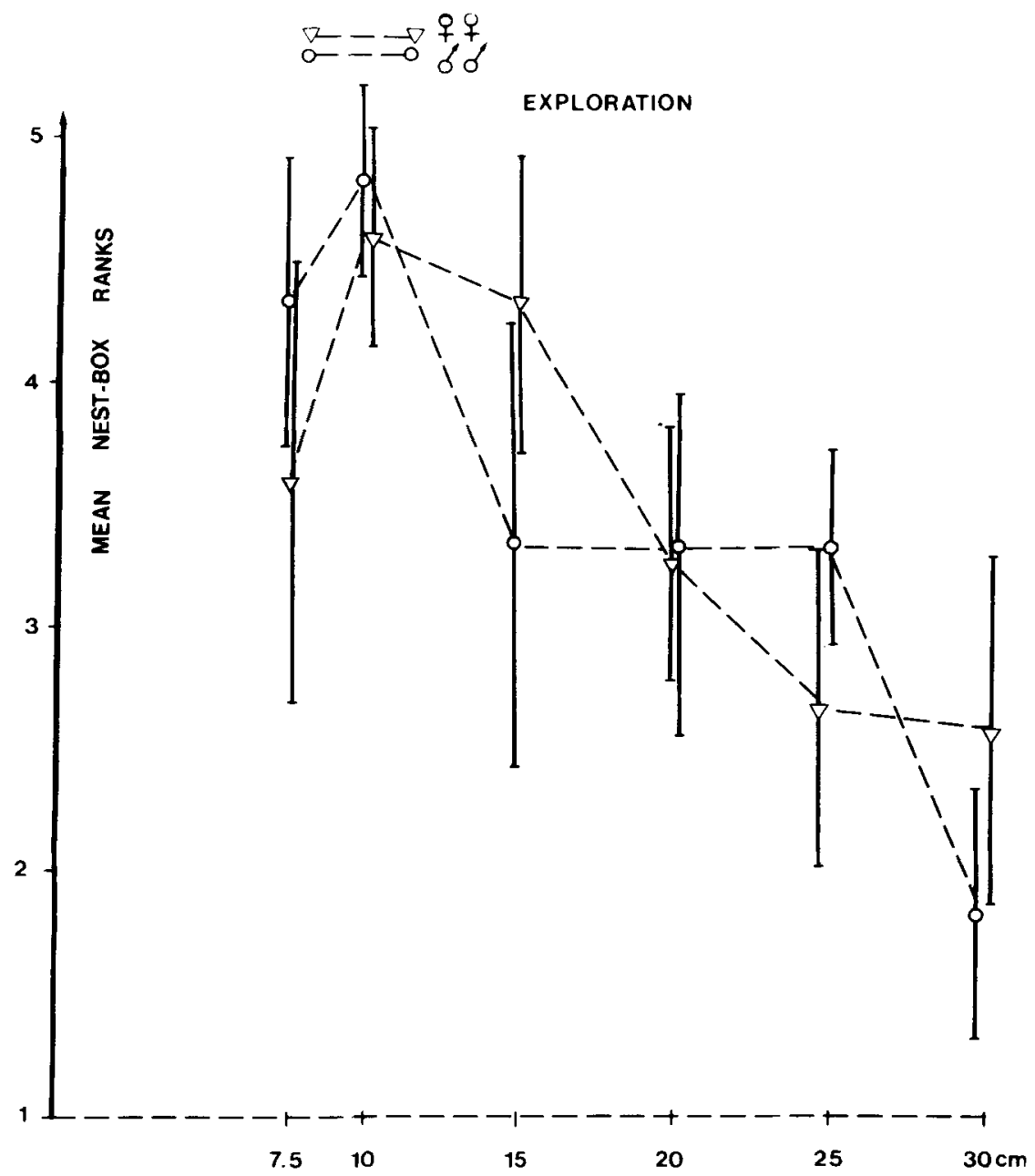

Figure 3. Nestbox exploration in male and female groups. Mean $( \pm S E M)$ ranks of the various nestboxes (diameter in centimeters on the horizontal axis).

with $d f 1=5$ and $d f 2=50, p<.05$ ). To test this finding statistically, a Wilcoxon matched sign test (Siegel, 1956) was applied to the difference between the sum of ranks allotted to the three smallest sized nestboxes and the sum of ranks allotted to the three largest ones. The result was significantly consistent with the finding of an overall effect of size on exploration $(T=8, N=12$, $p<.02$ ). With regard to the size of the groups, no significant correlation was observed between group size and size of the first nestboxes to be visited $(r=+0.08$, n.s.).

The exploratory patterns were further analyzed with regard to latency of initial visit to each nestbox. This measure is shown in Figure 4 for male and female groups. The latencies varied from $1 / 100 \mathrm{~min}$ to the $30 \mathrm{~min}$ at which the observation period ended (see Method). The 30-min value was allotted to the nestboxes that had not yet been explored during this time. The curves representing male and female groups show similar courses, with the shortest latencies being toward the largest nestboxes and conversely. The large standard error accounted for the differ- ence on the time scale in the interindividual variations. A Friedman two-way analysis of variance (Siegel, 1956) applied to these data showed that latencies increased inversely with the size of the nestboxes, but this result was not statistically significant (male and female groups pooled, $\left.\chi_{r}^{2}=10.12, d f=5, p<.10\right)$.

This analysis of initial exploration was concluded by considering the mean number of visits effected per individual during the 30-min observation period (see Figure 5). This figure again shows that distributions of visits to the nestboxes by male and female subjects were similar. Furthermore, it shows a connection between the increase in the mean number of visits and the increase in size of nestbox. These data were statistically analyzed using the Friedman two-way analysis of variance (Siegel, 1956), which showed the covariation of size/visits to have had a significant effect $\left(\chi_{r}^{2}=18.56, d f=5\right.$, $p<.01$ ). Otherwise, $47.25 \%$ of the total number of visits were made by males and $52.75 \%$ were made by females. But no sex-related differences were recorded in the ten- 


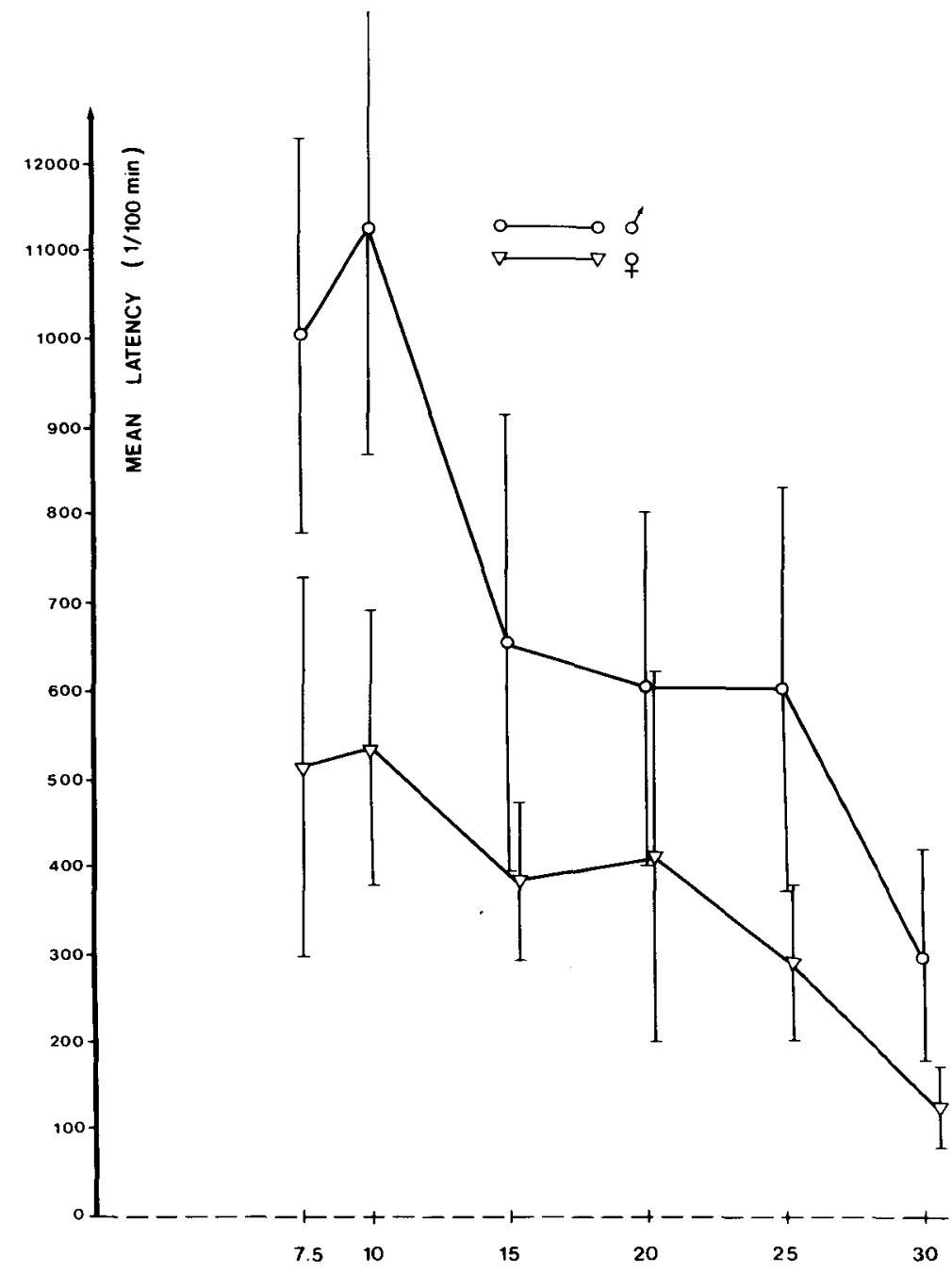

Figure 4. Nestbox exploration in male and female groups. Curves of mean $( \pm S E M)$ latency $(1 / 100$ min on the ordinate) to initial exploration of each of the six nestboxes.

dency to visit the largest nestboxes more frequently $(67 \%$ of the visits) than the smallest ones $\left(\chi^{2}=0.006, d f=1\right.$, n.s.).

With regard to the sizes of the groups, the percentage of the total number of visits made by groups of 2 or 3 subjects was $47 \%$, whereas that made by groups of 4 or 5 subjects was $53 \%$. Whatever the group size, a general tendency to visit larger nestboxes more frequently than smaller ones was observed; a difference was found, however, between the smaller ( 2 and 3 subjects) and the larger (4 and 5 subjects) groups, which visited the three largest nestboxes on $60.5 \%$ and $74 \%$ of the total number of visits, respectively $\left(\chi^{2}=20.16, d f=1, p<.001\right)$.

\section{Discussion}

The purpose of this initial experiment was to investigate systematically how metric parameters are perceived and used by mice in a spontaneous behavior, for example, in nest establishment. The use of such a continuous spatial parameter allowed a relevant comparison to be made between a preferential (i.e., "subjective") scale and a metric (i.e., "objective") one. This had not always been possible in our previous studies, in which shape was used as the main differentiating factor for the nestboxes $\mathrm{Bu}$ hot, 1986b; Buhot-Averseng, 1981). The systematic preference shown for a medium-small nestbox indicated that mice, rather than making absolute "judgments," tended to detect the relative differences between objects of various sizes. The elimination task was thus appropriate for use in studying the relative preferences, from the firstchosen nestbox to the least preferred (the remaining nestbox in the series); furthermore, it allowed for the occurrence, and hence the analysis, of a logical, or patent, rule of ranking. This ranking rule, or criterion, was further shown by the analysis of concordance between subjects (here the groups or the sexes) to be operational. The data 


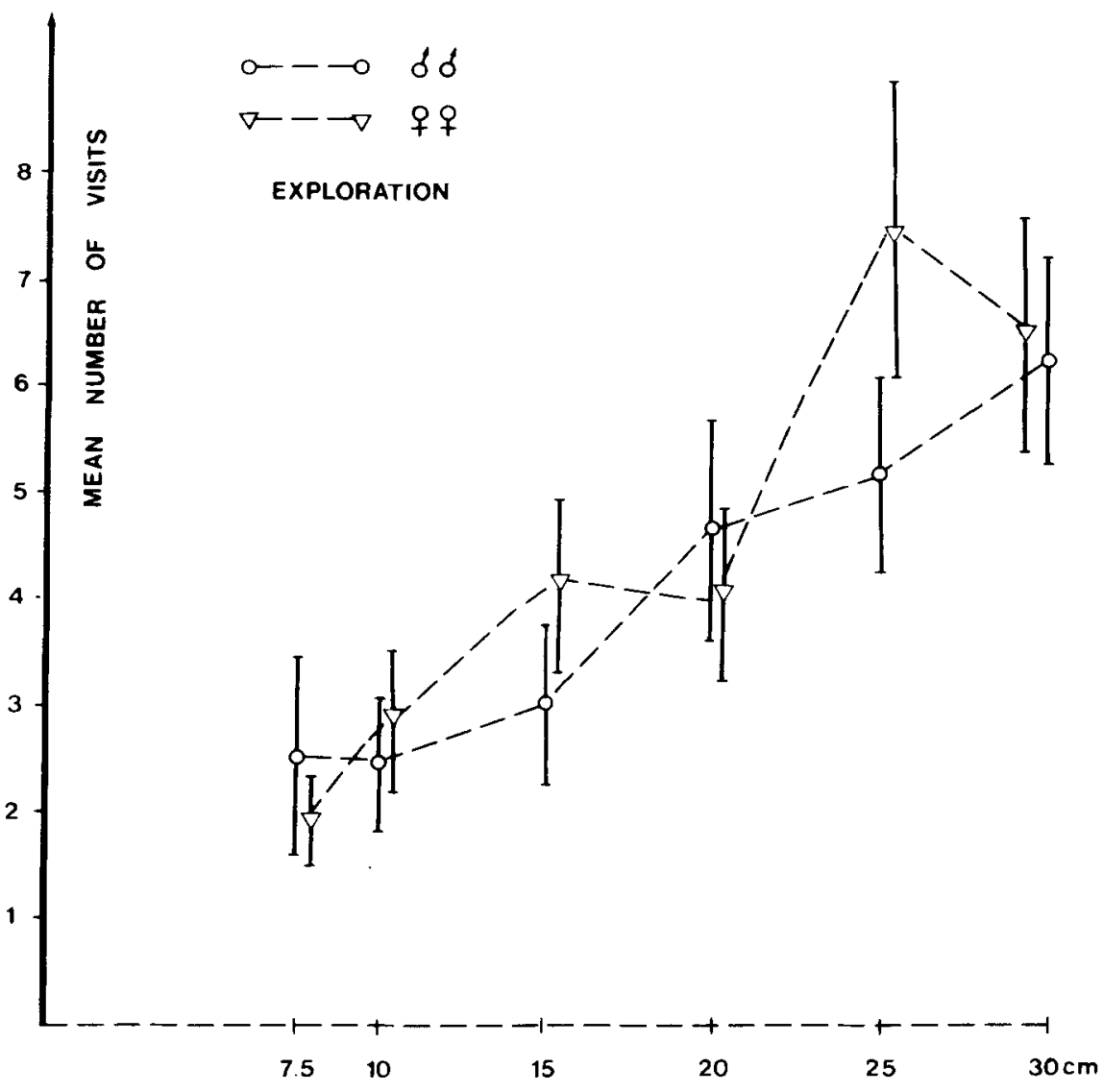

Figure 5. Nestbox exploration in male and female groups. Mean $( \pm S E M)$ number of visits per individual (on the ordinate) to each of the six nestboxes.

on nestbox choice thus showed that male and female groups' optimum choice of nestbox was concordant. With regard to exploration patterns, it was still more obvious that a common ranking criterion was applied by the subjects whatever their sex, but with regard to the size modalities, this criterion produced practically inverted preferences, as shown by analysis of the order latency and the number of visits: here the largest nestboxes were given preference. This exploration-guiding rule appears to be more general than the one accounting for nest-site choice, since it was applied whatever the size modalities, whereas the choice data showed an optimum and a decreasing preference toward smaller and larger nestboxes alternately. It may be that, after their first optimal choice, the mice hesitated between the next smallest and the next largest nestbox. They had to "decide" which of them was "the least undesirable," that is, which was the best among the remaining nestboxes. The distribution of the first choices was, in fact, responsible for the $U$ shape of the choice values, since four groups chose the $15-\mathrm{cm}$ nestbox first, three groups chose the $10-\mathrm{cm}$ box first, and three chose the $20-\mathrm{cm}$ box first; none chose the two largest $(25$ and $30 \mathrm{~cm}$ ) ones first. Furthermore, the second choices of the majority of the groups $(66 \%)$ were the 10 - and $15-\mathrm{cm}$ nest- boxes. This suggests that the first choice alone (which is the proper meaning of choice, i.e., selecting one among several objects) was concordant with the mean preferential ranking.

The tendency to visit larger nestboxes before smaller ones, and to more frequently visit the former than the latter confirms previous findings (Buhot, 1986b; BuhotAverseng, 1981). The reversal found in the patterns of use of size for exploratory and nestbox-choice purposes also confirms previous results. Since the conditions were such that it was assumed (Buhot-Averseng, 1981) that the large nestboxes were more likely to be encountered by the mice than the smaller ones during their exploratory travels (the former occupying a larger area in the field), a second experiment was planned to test both this hypothesis and the one described as follows. If nestbox choice and exploration are two behavioral processes that are negatively correlated in their use of size, it may be that the former (nest-site choice) is influenced by the inner characteristics of nestboxes that make a nestbox a more or less suitable shelter, and that the latter (exploration) is influenced by the outer characteristics of nestboxes that are more or less likely to be encountered (thus entered) during the initial phase of this choice process. 


\section{EXPERIMENT 2}

This second experiment was accordingly designed to establish how size influences nestbox exploration and nest establishment. More specifically, the effect of varying the inner parts of the objects while keeping their outer parts constant was examined. A second aim of this experiment was to analyze more closely the influence of the size of the group, since, in Experiment 1, in which male and female groups of different sizes ( 2 to 5 ) were used, the size of the group had not been observed to have any obvious effect. In the present study, only groups consisting of 2 or 3 male mice were used.

\section{Method}

Subjects. Twenty-four naive adult male mice (Mus musculus L.), aged about 3 months at the beginning of testing, were used in this experiment to form 10 same-sex weaning groups: 6 groups of 2 male mice and 4 groups of 3 male mice. They all came from the same stock as those used in Experiment 1, and were reared under the same standard conditions before and during the experiment as described previously.

Stimuli. Two sets of five nestboxes were used. The first set of nestboxes was composed of five simple nestboxes with different diameters $(5,10,15,20$, and $25 \mathrm{~cm})$; under this condition, the insides of the nestboxes were consistent with their outside appearance (matched condition). The second set of nestboxes consisted of five nestboxes with different inner diameters $(5,10,15,20$, and $25 \mathrm{~cm})$ but with the same outer diameter $(25 \mathrm{~cm})$; under this condition, the inner and outer dimensions were said to be mismatched. Like the stimuli used in Experiment 1, the nestboxes were removable and had a constant height $(8 \mathrm{~cm})$, no lids, and a 5-cm-wide aperture. The nestboxes that had smaller inner than outer diameters were fitted with a roof that covered the unavailable part between the inner (available) area and the outer limits of the boxes. All the nestboxes were made of sheet metal.

Test situation. Groups of mice were subjected to two progressive elimination, forced-ranking tests, as in Experiment 1, one with each of the two sets of nestboxes described above. All five nestboxes in a set were simultaneously available at the beginning of the test. The aperture of each nestbox was turned toward the center of the field; a square of nesting material (cellulose) with a bit of commercial food on top was placed in front of each nestbox. The subjects were placed by hand onto the middle of the field and were then observed while they explored the various nestboxes for $30 \mathrm{~min}$; the latency, the order of first visit to each box, and the number of visits made during this time were recorded. The mice were then left free until the first nestbox had been chosen as a nest site; that nestbox was then removed so that the mice had to move their nest to another box. This forced-elimination procedure continued until only one nestbox remained. The test order among the groups and the locations of the nestboxes during each test were set up to offset any order effects and position cues.

The set of nestboxes with identical outer limits (mismatched condition) was designed to neutralize any effects of the outside appearance of the nestboxes, since this factor had previously seemed to influence exploratory behavior. Our hypothesis was that with this set of nestboxes, the order of initial exploration would not reveal any size-related effects, since, from the outside, the five nestboxes appeared to be identical. With regard to nest-site choice, our hypothesis was that the results would not change, since the insides of the nestboxes, presumably the main factor in nest-site choice, varied as they had in Experiment 1. The five nestboxes in this mismatched condition also differed with regard to their inner sizes, as in the present matched (control) series.

\section{Results}

Data analysis. As in Experiment 1, the main data recorded using the elimination procedure were the ranks allotted by the subjects to the various nestboxes during initial exploration and during the nest-site elimination procedure. These data were also analyzed using ranking methods (Kendall's, 1962, coefficient of concordance; Buhot-Averseng, 1981, Appendix II). The effect of size was further analyzed using nonparametric statistical procedures (Siegel, 1956) applied to ranks and to the number of visits. Since the two experimental situations used differed in terms of whether the outer size of the nestboxes stood in a "matched" or a "mismatched" relationship to the inner size, the main results are illustrated by figures in which two curves represent each of these two situations.

Nestbox choice. The nestbox choice data, namely nestbox ranks, correspond to the successive choices made by the group considered as the entity (the subject) undergoing the test. In fact, the group's choice corresponds to a collective choice, since a common nest was generally built.

The preferences for the various nestboxes in both experimental situations are presented in Figure 6 . The two sets of objects are symbolized by the inner diameter along the horizontal axis. The preferred nestbox, which was given the optimum value (minimum mean rank), was the second smallest nestbox. The smallest nestbox was rejected (given the maximum mean rank), and then the decreasing order of preference followed the increasing order of (inner) size of the nestboxes. With regard to the two different sets of nestboxes corresponding to the two experimental conditions, matched and mismatched, no particular differences were observed. To measure the degree of agreement among the groups in ranking the nestboxes according to a common criterion, Kendall's (1962) coefficient of concordance was calculated on the rankings. In each experimental situation, the groups showed a significant concordance (matched-S $=772, W=0.772$, $\varrho=+0.746, z=1.71$, with $d f 1=4$ and $d f 2=34$, $p<.01$; mismatched $-S=350, W=0.35, \varrho=+0.278$, $z=0.79$ with $d f 1=4$ and $d f 2=34, p<.01$ ). To measure precisely the relationships between the order of preference and the order of inner sizes, the Friedman twoway analysis of variance was further applied to the ranks allotted with regard to their different inner areas. In the situation in which the inner and outer sizes varied equally (matched), the test indicated a strong order effect on the scale of size $\left(\chi_{r}^{2}=30.88, d f=4, p<.001\right)$. In the second situation (mismatched) using nestboxes with identical outer sizes, the results of the test again suggested that only the inner size affected the order $\left(\chi_{r}^{2}=14, d f=4\right.$, $p<.01$ ).

In another connection, the size of the groups did not appear to be a decisive factor in the choice of a nest site. In the matched condition, the first choices made by the 2 -mouse groups were all toward the 10 - and $15-\mathrm{cm}$ nestboxes (three groups preferred the 10 and three the 15); three of the 3-mouse groups chose the 10-cm nestbox and one chose the $15-\mathrm{cm}$ nestbox first. The correlation be- 


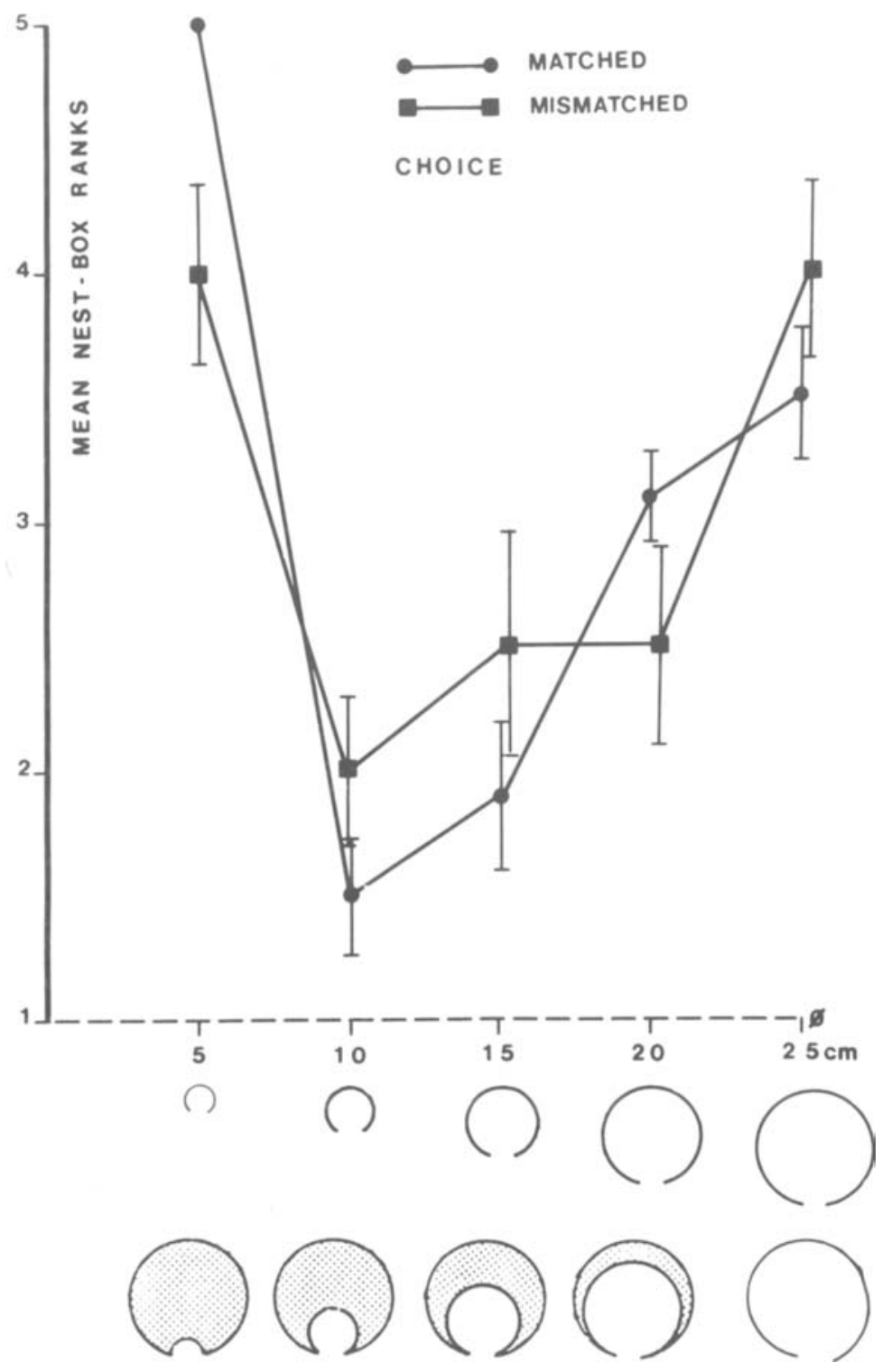

Figure 6. Nestbox choices. Mean $( \pm S E M)$ ranks of the five nestboxes differing in (at least) inner size (diameter in centimeters on the horizontal axis). The inner and outer dimensions of the nestboxes were either identical (matched) or not (mismatched).

tween the sizes of the groups and the sizes of the first nestboxes chosen was not significant $(r=+0.25$, n.s.). In the mismatched condition, the 3-mouse groups chose the $15-\mathrm{cm}$ and the $20-\mathrm{cm}$ nestboxes twice, and the 2 -mouse groups chose the $10-\mathrm{cm}$ nestboxes three times, the 15$\mathrm{cm}$ nestboxes twice, and the $20-\mathrm{cm}$ nestboxes once $(r=+0.53$, n.s.). On the basis of these tenuous differences, the size of the groups does not seem to have been a relevant factor.

Exploration patterns. As in Experiment 1, the exploratory behavior of the mice was observed during the first $30 \mathrm{~min}$ of the test. The analysis was again carried out on the ranks of the first visits to each of the nestboxes and on the number of visits to the various nestboxes during this 30 -min observation period.

As previously, the rank of the first visit to each nestbox corresponds to the first entry into each nestbox by any individual of the group. Figure 7 presents the mean nestbox ranks of first exploration in the matched and mismatched conditions. The effect of the size of the nestboxes can be seen in the matched condition, in which the larger nestboxes were usually visited before the smaller ones were. This effect was, however, limited to the extreme nestboxes on the scale of size. In the mismatched condition, the inner size (the only factor that varied) influenced the order of exploration far less, although a slight ten- 


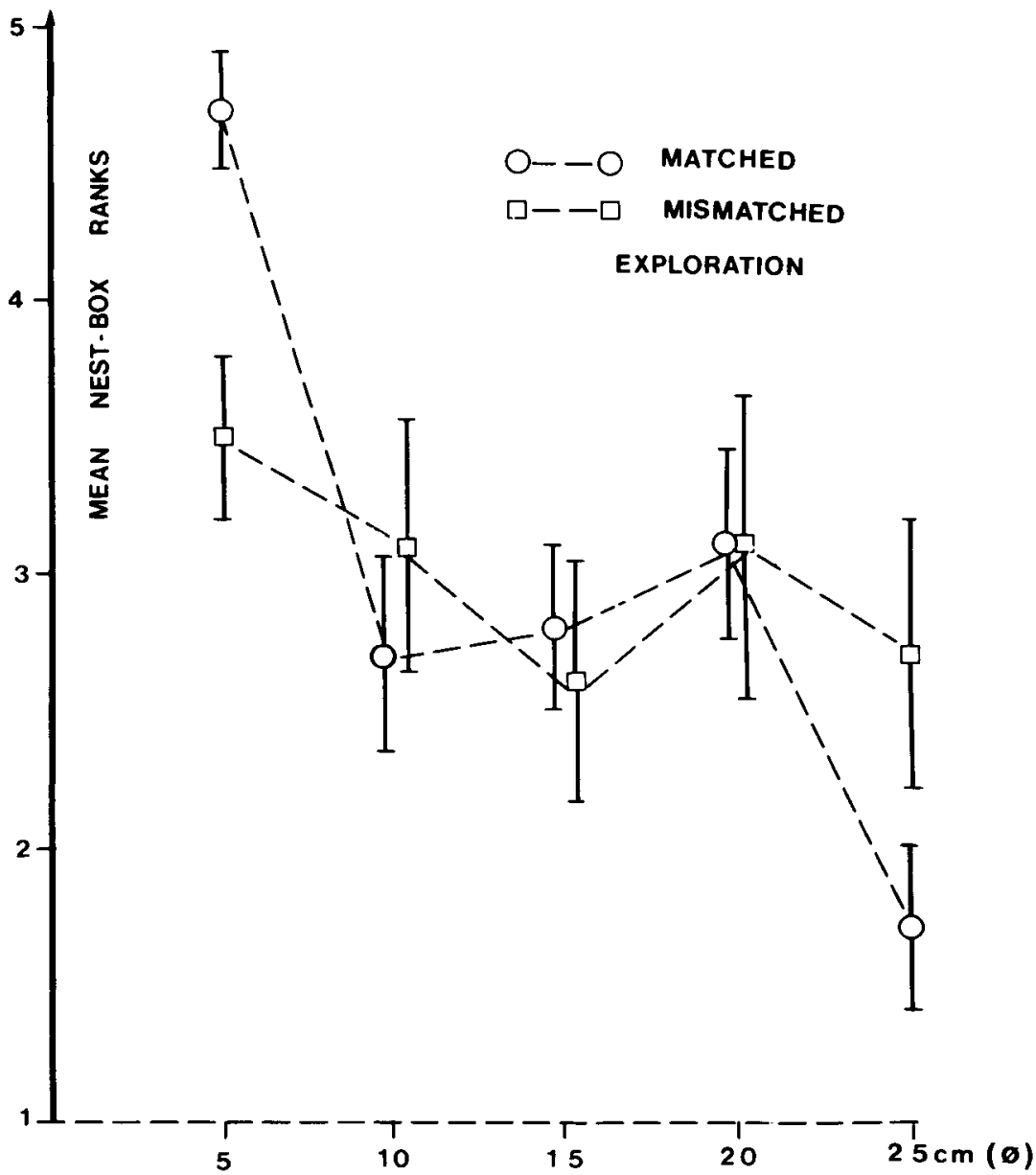

Figure 7. Nestbox exploration. Mean $( \pm S E M)$ ranks of the five nestboxes used in both tests. (For details, see Figure 6 caption.)

dency to explore the largest earlier than the smallest nestbox was observed. The concordance between groups was analyzed as previously with the choice data. In the matched condition, Kendall's coefficient of concordance showed that the groups were significantly in agreement in following a particular rule of exploration $(S=472$, $W=0.472, \varrho=+0.413, z=1.04$, with $d f 1=4$ and $d f 2=34, p<.01$ ); this was not the case in the mismatched condition, in which all the nestboxes were identical on the outside $(S=52, W=0.052, \varrho=0.05$, $z=0.35$ with $d f 1=4$ and $d f 2=34$, n.s.). To further determine the extent of these effects, Friedman's two-way analysis of variance was applied to the data. In the situation in which the inner and outer sizes of the nestboxes varied equally (matched condition), the results of this test were significant $\left(\chi_{r}^{2}=18.9, d f=4, p<.001\right)$, indicating that the groups' order of exploration was consistent with a systematic behavior. Conversely, in the mismatched condition, with nestboxes that looked identical from the outside, no significant effect of size was detected $\left(\chi_{r}^{2}=2.08, d f=4\right.$, n.s. $)$, which may indicate that the order of exploration was random.
Although a weak negative correlation was found between the size of the first-visited nestboxes and the size of the groups in the matched condition $(r=-0.56$, $p=.10)$, this was not the case in the mismatched condition $(r=+0.27$, n.s.).

The exploratory behavior was also studied from the point of view of the number of visits made to the various nestboxes during the initial $30 \mathrm{~min}$ of the observation period. Figure 8 presents, for both situations, the mean number of visits made per individual to the various nestboxes. The curves corresponding to the matched condition clearly followed the increasing order of size of the nestboxes; this pattern was far weaker in the mismatched condition. To confirm these findings statistically, the Friedman two-way analysis of variance was applied to the data. It was found that the systematic positive relationship between the number of visits and the size of the nestboxes was significant in the matched condition $\left(\chi_{r}^{2}=21.6, d f=4, p<.001\right)$ but not in the mismatched condition $\left(\chi_{r}^{2}=5.14, d f=4\right.$, n.s. $)$.

With regard to the group size, $52.4 \%$ of the visits were made by groups of 3 mice and $47.6 \%$ were made by 


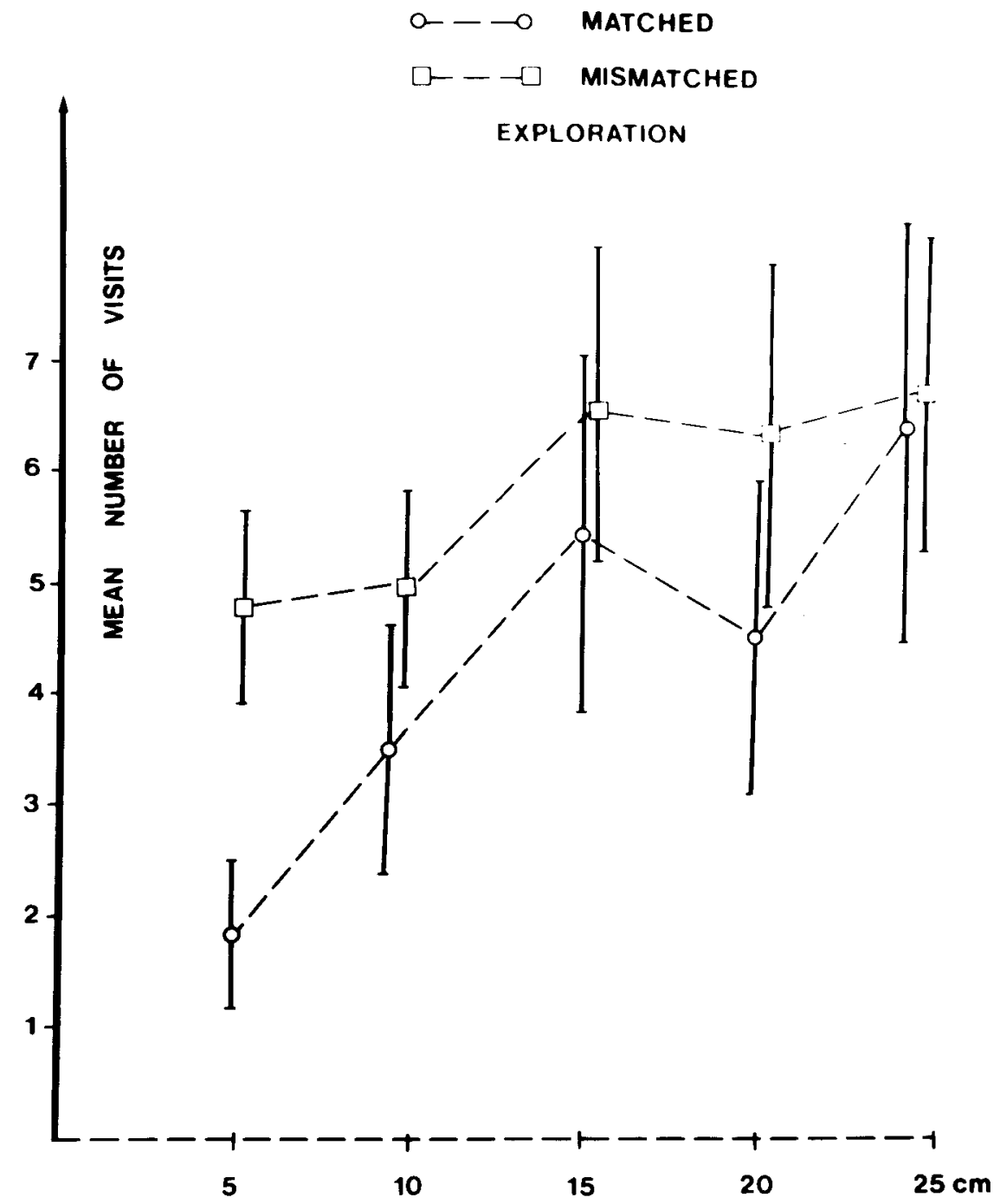

Figure 8. Nestbox exploration. Mean $( \pm S E M)$ number of visits per individual (on the ordinate) to each of the five nestboxes used in both tests. (For details, see Figure 6 caption.)

groups of 2 mice in the matched condition; these percentages were $55 \%$ and $45 \%$, respectively, in the mismatched condition. With regard to the visits made to the various nestboxes, a significant difference between groups of 3 and 2 mice was observed in the matched condition $\left(\chi^{2}=\right.$ $18, d f=4, p<.001)$, but not in the mismatched condition $\left(\chi^{2}=5.2, d f=4\right.$, n.s.). However, in the matched condition, groups of both sizes tended to visit the larger nestboxes more frequently than they did the smaller nestboxes, since $67 \%$ of the visits made by groups of 3 mice and $60 \%$ of the visits made by groups of 2 mice were to the two larger nestboxes. When tested by a chi-square test, this difference, including the size of the nestboxes and the size of the groups, was found to be nonsignificant $\left(\chi^{2}=0.19, d f=1\right)$.

\section{Discussion}

The second experiment was designed to further study whether the size of the nestboxes was an important fac- tor in the choice of nest site, and whether it might also influence the initial exploratory patterns.

In the first study conducted along these lines (Experiment 1), six simple nestboxes were used; they differed only in size, and their inside areas were the same as those delimited by their outside walls. These objects were similar to those used here in the matched condition of Experiment 2, except that the smallest was $7.5 \mathrm{~cm}$ in diameter and the largest, $30 \mathrm{~cm}$ in diameter. The results showed that the preferences of male and female groups were similar, favoring smaller rather than larger nestboxes in an order based on their sizes: the larger they were, the earlier they were visited. These results were confirmed in Experiment 2 with a set of slightly different nestboxes. It is particularly interesting to note that the mice's reaction toward the nestboxes was not based on a principle of "absolute" preference for the smallest nestboxes, since in both Experiment 1 and Experiment 2, an optimum preference for a medium-small nestbox was found. The rela- 
tivity of the optimum value needs to be emphasized. In Experiment 1, the 15-cm-diam nestbox was the most frequently preferred among a set that included the two extreme sizes -7.5 and $30 \mathrm{~cm}$ in diameter. In the present experiment, with a range of sizes from 5 to $25 \mathrm{~cm}$ in diameter in steps of $5 \mathrm{~cm}$, the optimum value was $10 \mathrm{~cm}$. The procedure used to induce preferential responses may account for this difference. The elimination procedure indeed induced the animals to "evaluate" the respective advantages of all the remaining nestboxes by comparing them before each successive choice. This operation may be dependent upon the relative sizes of the available objects.

A new situation (mismatched) was further designed to neutralize the outside appearance of the nestboxes, since this factor was presumed (Experiment 1) to influence exploratory behavior. The results of the present experiments showed that the inside dimensions of the nestboxes was the main factor in nest establishment, since no obvious difference in nest-site choice was observed between the matched and mismatched conditions. However, the exploratory patterns found in the mismatched condition were in fact "disturbed" in relation to the control (matched) situation, since no systematic patterns of exploration (i.e., obeying some rule relating to size) were observed in this new situation. We therefore conclude that the main role in exploration is played on the basis of the outer characteristics of the nestboxes, since a lack of difference between the nestboxes on the outside led to a disorganization of the exploration dynamics.

\section{GENERAL DISCUSSION}

The present study focused on the preferential use by mice of subspaces, that is, nestboxes usable as nest holders. These boxes differed only with regard to size.

The results thus extend our previous tenuous findings on nestbox choice, since, on average, the smaller nestboxes were chosen before larger ones. The present original findings were that this trend was not linear or absolute, but provided scales around an optimum value like a gradient of generalization. These data did not otherwise demonstrate any sex-related effect or a tendency in the groups to adapt to the situation by choosing preferentially the nestboxes that were the most suitable in size for the size of the group. The majority of the groups first chose a nestbox from among the smaller ones; the two largest nestboxes were never used as the first nest site. It is likely that this tendency to establish the nest in a limited space has to do with the well-known gregariousness of mice and rats (see Cairn, 1966; Ewer, 1968; Latané, 1969). Furthermore, the experimental situation, which involved the cagemates' being placed in a large, unknown field, may have been to some extent fear-inducing, even if this social condition was not as stressing as isolation. The animals were thereby induced to search for closeness among themselves by choosing as a nest site the most convenient (narrow) available walled portion of space.
Results quite similar to those of Experiment 2 were obtained by Buhot (unpublished data), who used the same set of objects but a different procedure, that is, successive pair comparisons. This procedure precludes the animal from simultaneously comparing each object with all others of the set. When only two objects are present at a time, the relative choice is limited to an alternative comparison. The fact that no difference in the preferential scale was obtained, using both procedures, might indicate that the animals showed consistent preferences whatever the number of choice alternatives.

Nevertheless, it could also be useful to use other ranges of nest-site sizes to determine more precisely whether mice respond to actual or relative size cues. Another way is to study independently the interest of the animal for each of the nestboxes, presented one at a time, by taking, for instance, the latency to explore and habituate and finally to use (or not) as nest site. In fact, in certain conditions, when, for example, the subject is left in presence of the last remaining nestbox, one can observe either the use of this box as nest site or not (the nest being then simply moved by the mouse to the wall of the open field). This lack of choice or avoidance can be defined as an absolute choice, but its occurrence depends on the contextual condition, which, in the present example, gives no opportunity for the subject to "prefer" this remaining nestbox to (nonpresent) others which could here have been still "worse."

In another connection, the motivation of the mouse to establish its nest in a particular place depends on the time the mouse spends outside the home cage and the availability of nest material, food, and water. This condition is sufficient to allow for the occurrence of nest establishment. Since the room was maintained at a constant temperature, and since nest building occurred whatever the season or outside temperature, it is not likely that weather played any role in the nest building. Nevertheless, it may be that the relative properties of the boxes (e.g., the constituent material-metal or Plexiglas-or, here, the narrowness) could have played a role in thermoregulation. This could explain the observed tendency to choose in terms of priority the smaller nestboxes.

The results showed an average negative correlation in the preferential scale of size of the nestboxes between the initial exploratory behavior and the "concluding" successive nest-site choices, that is, between the tendency to explore first the larger nestboxes and the tendency to choose first the smaller ones as nest sites.

This apparent incongruity can probably be explained by the fact that exploration and nest establishment do not have the same "cost" for the animal. The first class of behavior is included in the dynamic of spatial knowledge in a new, unknown environment. The second behavior, nest establishment, might provide for the welfare of the animal itself: thermoregulation and search for comfort and shelter. Exploration, which is the very first phase of this choice long-term behavior, is a short-term process, which, in time, decreases with habituation. The larger the nestboxes were, the earlier and more frequently they were 
explored. We have discussed the hypothesis, taking into account that, in case of random locomotor activities, the probability of encountering the boxes increases with increases in the sizes of the objects. On the other hand, nestsite choice requires a long-lasting stabilization of the animal in the experimental environment.

This procedure of allowing the occurrence of spontaneous choices is a particular way of studying animals' knowledge of space. To some extent, it can be said to be a psychophysical approach (Thinus-Blanc \& BuhotAverseng, 1985, and, in human psychology, WienerEhrlich, 1978), since a demonstrated preference means that the subject has discriminated among the nestboxes (or three-dimensional stimuli) on the basis of the parameter(s) that define their differences. This constitutes our main purpose, that is, to provide information about the perceptual abilities of mice using natural motivation under an experimentally contrived situation.

This recalls the very first studies to be devoted to animal discrimination learning, particularly the role of relative versus absolute cues. Lashley (1912) found, for example, that rats trained in size discrimination reacted to relationships between the (plane) stimuli. Kohler (1929) suggested that animals learned more easily to react to relationships between stimuli than to absolute cues (i.e., particular stimuli). Gulliksen (1932) suggested two hypotheses that could also account for our data. He suggested, first, that some animals primarily learned to avoid a negative stimulus, whereas others learned mainly to seek a positive stimulus. Our experimental elimination paradigm causes animals to make each choice among the remaining boxes a positive one; simultaneously, it encourages the animals to avoid (i.e., to reject or simply to neglect) the less suitable ones. Gulliksen also suggested that a transfer of response was mainly confined to a limited range around an absolute size. This hypothesis, which accounts for discrimination learning data, is consistent with our findings of a linear relationship between size and preference convergent upon the optimum value. Weiss, Ernst, and Schick (1982), in studying rats' preferences for cages of different sizes, obtained other results that agreed with this rule.

Most basic research on animals' knowledge of space has been approached by using learning paradigms, such as discrimination learning (Sutherland, 1964, 1968), primarily among two-dimensional stimuli (e.g., Dodwell, 1960; Zimmermann, 1962; Zusne, 1970). A wide range of species has been investigated in this way (see, e.g., Ingle, 1978; Sutherland, 1961, 1968; Thinus-Blanc, 1975).

Concerning the ability of mice to differentiate in terms of shape and size, only a few, old studies have been devoted to the discrimination of (mainly) plane figures. Rowley and Bolles (1935) showed that white mice could discriminate between geometrical figures with equal areas, and Worner (1936) provided some evidence that gray mice were able to transfer a shape discrimination along a continuum using shapes that were intermediate between two different ones. Boxberger (1952) confirmed the existence of shape-discrimination ability in mice and rats, and Sloane, Shea, Procter, and Dewsbury (1978) showed that house mice were able to discriminate depth.

Object, or volume, discrimination has been studied even more rarely. Thinus-Blanc (1978) showed that such small mammals as the golden hamster were able to discriminate between two cubic volumes of different sizes and that in test situations they attended mainly to width, one of the three dimensions that defined the volume. Furthermore, Thinus-Blanc (1981) showed that this ability depended on rearing conditions, since subjects reared in spatially diversified cages were influenced by depth as well as width. In other words, the "enriched" animals were able to take into account the surfaces of volumes, whereas standard subjects used only one dimension-width.

Another, more recent line of research on how animals perceive space has included studies on object exploration. An interesting experimental paradigm (Poucet, Chapuis, Durup, \& Thinus-Blanc, 1986) consisted of giving animals (hamsters) the opportunity to explore various objects arranged in a particular way in an open field. In a second phase of exploration, this investigatory activity decreased as a result of habituation. A change introduced before the last session of exploration (for example, two objects might be interchanged or only one displaced) generally caused a renewal of exploration (mainly toward the moved object[s]) in the final session. This seems to indicate that the animals perceived the change (the study in question dealt with the use of absolute or relative position cues). Further investigation along these lines, introducing changes in the characteristics (size, shape, etc.) of objects, might usefully extend our knowledge of animal perception, attention, and cognition with regard to the spatial features of the fixed points or landmarks within the environment.

\section{REFERENCES}

BoxberGer, F. v. (1952). Vergleichende Untersuchungen über das visuelle Lernervermögen bei weissen Ratten und weissen Mausen. Zeitschrift für Tierpsychologie, 9, 433-451.

Buнот, M.-C. (1986a). Espace subjectif, espace objectif. Comportements de choix chez l'animal. Résultats expérimentaux. Perspectives comportementales et neurobiologiques. Thèse de Doctorat d'Etat, Université d'Aix-Marseille II.

BUнOT, M.-C. (1986b). Nest-box exploration and choice in male and female mice tested under individual and social conditions. Behavioural Processes, 13, 119-148.

Buhot-Averseng, M.-C. (1981). Nest-box choice in the laboratory mouse: Preferences for nest-boxes differing in design (size and/or shape) and composition. Behavioural Processes, 6, 337-384.

Calrns, R. B. (1966). Attachment behavior of mammals. Psychological Review, 73, 409-426.

DODWELl, P. C. (1960). Discrimination of small shapes in the rat. Quarterly Journal of Experimental Psychology, 12, 237-242.

EWER, R. F. (1968). Ethology of mammals. New York: Plenum Press. Firtner Kinder, E. (1927). A study of the nest-building activity of the albino rat. Journal of Experimental Zoology, 47, 117-161.

GULLIKSEN, H. (1932). Studies of transfer of response: I. Relative versus absolute factors in the discrimination of size by the white rat. Journal of Genetic Psychology, 40, 37-51. 
INGLE, D. (1978). Mechanisms of shape-recognition among vertebrates. In R. Held, H. W. Leibowitz, \& H.-L. Teuber (Eds.), Handbook of sensory physiology: Vol. 8. Perception (pp. 259-287). Berlin: Springer-Verlag.

KENDALL, M. G. (1962). Rank correlation methods (3rd ed.). London: Griffin.

KOHLER, W. (1929). Gestalt psychology. New York: Liveright.

LASHLEY, K. S. (1912). Visual discrimination of size and form in the albino rat. Journal of Animal Behavior, 2, 310-331.

LATANÉ, B. (1969). Gregariousness and fear in laboratory rats. Journal of Experimental Social Psychology, 5, 61-69.

Lisk, R. D., Pretlow, R. A., \& Friedman, S. M. (1969). Hormonal stimulation necessary for elicitation of maternal nest-building in the mouse (Mus musculus). Animal Behaviour, 17, 730-737.

LYNCH, C. B. (1973). Environmental modification of nest-building in the white-footed mouse, Peromyscus leucopus. Animal Behaviour, 22, 405-409.

Mulder, J. B. (1975). Bedding preferences of pregnant laboratoryreared mice. Behavior Research Methods \& Instrumentation, 7, 21-22.

Poucet, B., Chapuis, N., Durup, M., \& Thinus-Blanc, C. (1986). A study of exploratory behavior as an index of spatial knowledge in hamsters. Animal Learning \& Behavior, 14, 93-100.

Rowley, J. B., Bolles, M. M. (1935). Form discrimination in white mice. Journal of Comparative Psychology, 20, 205-210.

SIEGEL, S. (1956). Non-parametric statistics for the behavioral sciences. New York: McGraw-Hill.

Sloane, S. A., Shea, S. L., Procter, M. M., \& Dewsbury, D. A (1978). Visual cliff performance in 10 species of muroid rodents. Animal Learning \& Behavior, 6, 244-248.

SuTHERLAND, N. S. (1961). The methods and findings of experiments on the visual discrimination of shape by animals. Experimental Psychology Monograph No. 1, 1-68.
Sutherland, N. S. (1964). The learning of discrimination by animals. Endeavour, 23, 148-152.

SutherLAND, N. S. (1968). Outlines of a theory of pattern recognition in animals and man. Proceedings of the Royal Society, B, 171, 297-317.

Thinus-Blanc, C. (1975). Données comportementales sur la discrimination visuelle chez l'animal. Année Psychologique, 75, 457-491.

Thinus-Blanc, C. (1978). Volume discrimination in golden hamsters. Perceptual \& Motor Skills, 46, 1007-1013.

Thinus-Blanc, C. (1981). Volume discrimination learning in golden hamsters: Effects of the structure of complex rearing cages. Developmental Psychobiology, 14, 397-403.

Thinus-Blanc, C., \& Buhot-Averseng, M.-C. (1985). A basic approach of space-behaviour relationships in mammals. In $\mathrm{R}$. Zayan (Ed.), Social space for domestic animals (pp. 239-261). Dordrecht: Martinus Nijhoff Publishers.

Weiss, J. v., ERNST, A., \& SCHICK, K. L. (1982). Wahlverhalten als Beurteilungs Kriterium für die Haltungsbedingungen von Laborratten. Zeitschrift für Versuchtierkunde, 24, 193-201.

WIENER-EhrLich, W. K. (1978). Dimensional and metric structures in multidimensional stimuli. Perception \& Psychophysics, 24, 399-414.

WORNER, R. (1936). Über die Leistungsgrenze beim Auffassen figuraler Gestalten durch Mause. Biologische Zentralblatt, 56, 2-27.

ZimMERMANN, R. R. (1962). Form generalization in the infant monkey. Journal of Comparative \& Physiological Psychology, 55, 918-923.

Zusne, L. (1970). Visual perception of form. New York: Academic Press.

(Manuscript received May 12, 1986; revision accepted for publication March 27, 1987.) 